\title{
Pemphigoid gestationis
}

\author{
Céline M J G Lardenoije, Marije van de Water, Helena J M M Mertens, Ed T C M Gondrie
}

Department of Obstetrics and Gynaecology, Orbis Medical Centre, Sittard, The Netherlands

Correspondence to Céline M J G Lardenoije, c.lardenoije@orbisconcern.nl

\section{Summary}

A 39-year-old woman in the 39th week of her fifth pregnancy presented with severe itching. In recent weeks she had developed an increasingly itchy rash on her trunk and arms. Upon examination we observed multiple erythematous plaques and vesicles. Histological examinations of two skin biopsies confirmed the diagnosis of pemphigoid gestationis. Because the patient's complaints worsened we decided to deliver the baby, and as it was in transverse lie this was by caesarean section. A healthy son without rash was born. Although pemphigoid gestationis occurs in only 1 in 50000 pregnancies, it is very important to be aware of this condition when a pregnant woman has itching. Not recognising pemphigoid gestationis may lead to inadequate maternal treatment and possible preterm birth and neonatal pemphigoid gestationis.

\section{BACKGROUND}

Pemphigoid gestationis is a rare but important cause of itching in pregnant women. Given that itching is a common and often harmless complaint in pregnancy, it is often ignored (obstetric cholestasis, which causes itching without rash, may similarly be missed). If general practitioners and other specialists are not aware of this condition, the pregnant woman will not receive adequate treatment, possibly leading to preterm birth and neonatal pemphigoid gestationis. The aim of this communication is to increase awareness among clinicians of the diagnosis of pemphigoid gestationis in a pregnant woman with itching.

\section{CASE PRESENTATION}

A 39-year-old woman in her fifth pregnancy presented to the obstetrics department with severe itching.

The obstetric history showed four uneventful pregnancies and deliveries. At 39 weeks' gestation, the patient suddenly developed an increasingly itchy rash over her whole trunk which spread to the arms (figure 1). She did not have fever or feel ill. There was no history of allergic reactions. Triamcinolone, lidocaine and menthol cream had been given to the patient by her general practitioner, but all were ineffective.

\section{INVESTIGATIONS}

On physical examination we observed a pregnant woman with a fundal height according to a gestational age of 39 weeks. The fetus was in good condition. We observed multiple erythematous plaques and vesicles over the entire trunk and arms (figure 2). There were no blisters or pustules. Laboratory examination did not show any abnormalities (no eosinophilia, and normal infectious parameters, liver function and bilirubin).

Abdominal ultrasound showed a normal fetus lying in a transverse position. Two skin biopsies, one of the arm and one of the trunk, were performed by a dermatologist.

Histological examination confirmed the diagnosis of pemphigoid gestationis.

\section{DIFFERENTIAL DIAGNOSIS}

- pemphigoid gestationis

- erythema exsudativum multiforme.

\section{TREATMENT}

Because the patient's complaints worsened we decided to deliver the baby, by caesarean section as it was in transverse lie. A healthy son of $3380 \mathrm{~g}$ without rash was born.

\section{OUTCOME AND FOLLOW-UP}

After the baby was born, all skin lesions disappeared in a couple of days. Afterwards the patient had no further complaints.

\section{DISCUSSION}

Pemphigoid gestationis, previously referred to as herpes gestationis, is a rare autoimmune disease of pregnancy and the postpartum period. Despite its name, herpes gestationis is not related to herpes virus infections (the old term reflects the occurrence of herpetiform lesions). Pemphigoid gestationis occurs in approximately 1 in 50000 pregnancies, ${ }^{1}$ and characteristically appears during the second or third trimester. In rare cases, it starts in the first trimester or in the postpartum period.

Pemphigoid gestationis may occur in association with other autoimmune diseases, trophoblastic tumours, hydatiform mole and choriocarcinoma. ${ }^{2-5}$ Women with a history of pemphigoid gestationis are at higher risk of developing Graves' disease. ${ }^{2} 6$

The aetiology of pemphigoid gestationis is not completely understood. It is presumed that auto-antibodies are formed against the placenta. These auto-antibodies then cross-react with an antigen on the basement membrane of the skin. This immune response is mainly directed towards the bullous pemphigoid antigen with a molecular weight of $180 \mathrm{kDa}$, bp $180 .^{78}$ A lesser immune response is also directed against bp 280. ${ }^{9}$ Blistering of the skin occurs as a consequence of this autoimmune reaction.

The reaction of antibodies on the skin is identical to the reaction seen in parapemphigus. 


\section{BMJ Case Reports}

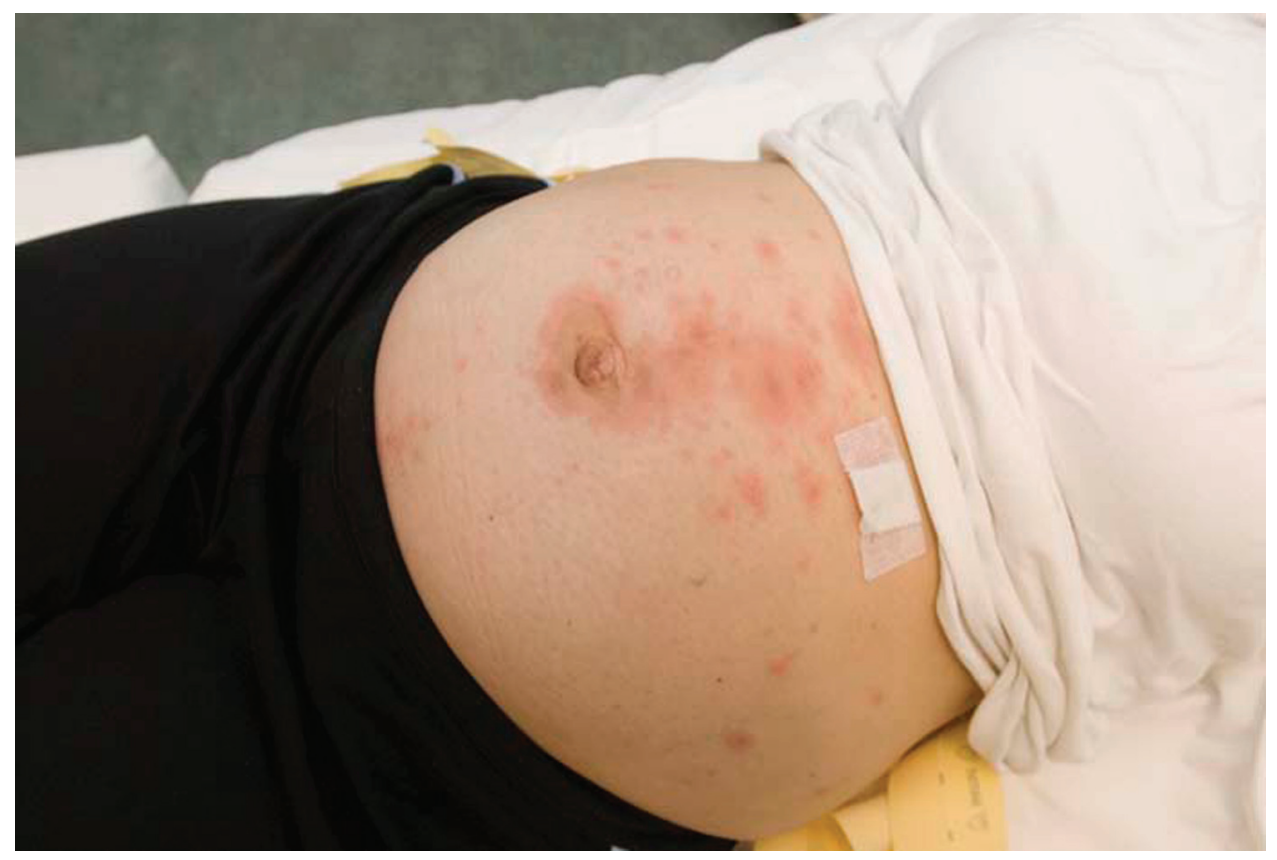

Figure 1 Pemphigoid gestationis on the abdomen.

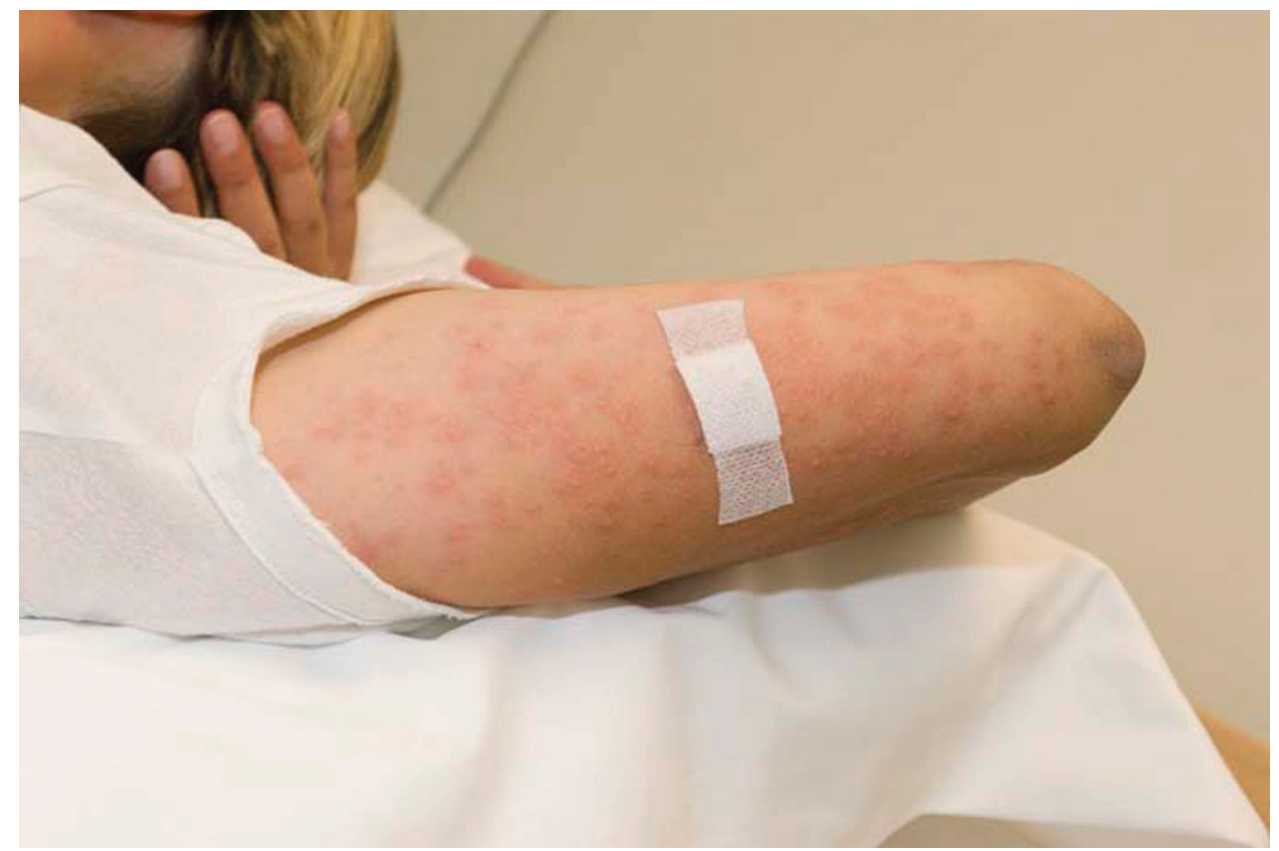

Figure 2 Pemphigoid gestationis on the arms.

Pemphigoid gestationis usually starts with intense itchy erythematous papules and plaques around the umbilicus which quickly spread to the abdomen, back, chest and extremities, sparing the face, mucosal surfaces, palms and soles. ${ }^{61011}$ Later, blisters filled with clear liquid are formed, although in some cases blisters may be absent. ${ }^{12}$

Classic histopathological findings in pemphigoid gestationis are subepidermal vesicles, with lymphocytes and eosinophils in a perivascular distribution within the dermis. The presence of eosinophils is the most constant feature of the histopathological appearance of pemphigoid gestationis. ${ }^{13} 14$
Symptoms in the early stage of the condition can be similar to those of pruritic urticarial papules and plaques of pregnancy (PUPP), which is a more common cause of itching during pregnancy. ${ }^{15}$ PUPP differs from pemphigoid gestationis in that it lacks both blisters and an autoimmune response.

The diagnosis of pemphigoid gestationis can be made from the general clinical image and can be confirmed by immunoblotting or ELISA, both of which are sensitive methods to prove auto-antibodies against bp 180. Investigations showed that in patients with pemphigoid gestationis auto-antibodies against bp 180 were detected 
in $93 \%$ of cases by immunoblotting and in $86.3 \%$ by ELISA. ${ }^{16}$ The severity of the disease may be correlated with the auto-antibodies detected by ELISA and/or eosinophilic leukocytosis. ${ }^{16} 17$

The typical finding at immunofluorescence is the C3 complement component, and in $25 \%$ immunoglobulin G (IgG) in a linear band along the basement membrane. ${ }^{13}$ 18-21

Pathophysiologically, the antibodies bind to the basement membrane which causes a complement activation through the classical complement pathway. Chemoattraction of eosinophils and their subsequent degranulation then take place. ${ }^{13} 22$ This presumably happens due to the release of proteolytic enzymes from eosinophilic granules that dissolves the bond between the dermis and epidermis. ${ }^{13}$

An IgG serum antibody is almost always found by indirect immunofluorescence, even when IgG is not seen by direct immunofluorescence. The circulating IgG antibody which fixes complement at the basement membrane is designated the herpes gestationis factor. ${ }^{13}$ 18-20 23

Immunogenetic examination has shown an increase in the HLA antigens DR3 and DR4 in patients with pemphigoid gestationis: $61-80 \%$ have DR3, $52-53 \%$ have DR4 and $43-50 \%$ have both. ${ }^{6} 24$

Treatment of pemphigoid gestationis starts with application of topical corticosteroids and/or lotions to decrease itch. Antihistamines can also help to control itching. In patients with severe symptoms, systemic steroid therapy is needed..$^{25}$ If these treatments are ineffective, plasmapheresis, cyclophosphamide, ciclosporin and dapsone are reported to be useful. ${ }^{26} 27$ However, their side-effects harm the mother and child. ${ }^{28} 29$

Generally, pemphigoid gestationis tends to exacerbate after birth (75\% of patients flare post partum). ${ }^{13}$ The duration of active disease ranges from 2 weeks post partum to 12 years post partum. ${ }^{10}$ In addition, some women experience recurrence of disease when taking oral contraceptives or during menses. ${ }^{17} 233031$ In one study recurrence of pemphigoid gestationis was found in $12 \%$ of women during menstruation. ${ }^{17}$

The possibility that pemphigoid gestationis will recur again in a subsequent pregnancy is high at almost $90 \%$, and usually occurs earlier in pregnancy and is more severe. ${ }^{13}$ Sometimes it may skip pregnancies. ${ }^{10}$

There is no consensus about the influence of a new partner on the risk of pemphigoid gestationis in following pregnancies. Some reports describe reducing prevalence with a new partner, while others find no differences. ${ }^{1} 10$

Pemphigoid gestationis should be considered as an indication for a clinical delivery.

Approximately $10 \%$ of all neonates of mothers with pemphigoid gestationis have cutaneous lesions similar to pemphigoid gestationis. ${ }^{13}$ The disease in children seems to originate from passive transfer of the pemphigoid antibodies, but could also be caused by resting maternal hormones. ${ }^{1}$ The skin lesions resolve spontaneously in the first months. ${ }^{32}$ Immunofluorescence of the newborn skin may be positive, even when there are no clinical symptoms of the disease. ${ }^{18} 3334$ When high doses of corticosteroids are given during pregnancy, the newborn baby must be examined immediately after delivery. Adrenal insufficiency has to be excluded, especially if the mother has used corticosteroids for a long period. ${ }^{35}$ Besides, dapsone may cause haemolytic disease in the child. ${ }^{36}$ Whether pemphigoid gestationis itself leads to complications in the child is still unclear.

Study of neonatal complications showed that there was morbidity or mortality in $38 \%$ of cases of pemphigoid gestationis: premature partus in $23 \%$, neonatal herpes gestationis in $9 \%$ and intrauterine death in $7.7 \% .{ }^{17} \mathrm{~A}$ relationship between pemphigoid gestationis and prematurity was also found in other studies. ${ }^{2} 37$ Shornick and Black showed that $16 \%$ of pregnancies associated with pemphigoid gestationis ended before 36 weeks and $32 \%$ before 38 weeks, compared to $2 \%$ and $11 \%$ in uncomplicated pregnancies. ${ }^{2}$ Further, a link was shown between pemphigoid gestationis and lower birth weight. This could be a consequence of mild placental insufficiency through an immune response between placental antigens and antibodies against the skin. ${ }^{38}$ Shornick and Black did not find an increase in spontaneous abortions or stillbirths in patients with pemphigoid gestationis, nor did the use of systemic steroids appear to influence the risk of preterm birth or lower birth weight. ${ }^{39}$

There is no evidence in the literature that pregnancy should be ended because of neonatal risks, likely due to the fact that most studies do not find an elevated risk of stillbirth. We do not think it necessary to induce labour, but advise close monitoring by regular ultrasound in case of low birth weight. Furthermore, it is important to be aware of the risk of preterm birth.

\section{Learning points}

- Be aware of the possibility of pemphigoid gestationis in a pregnant woman with itching.

- A pregnant woman with pemphigoid gestationis should be treated in hospital, and closely monitored because of the elevated risk of preterm birth and low birth weight.

\section{Competing interests None}

Patient consent Obtained.

\section{REFERENCES}

1. Shornick JK, Bangert JL, Freeman RG, et al. Herpes gestationis: clinical and histologic features of twenty-eight cases. J Am Acad Dermatol 1983:8:214-24

2. Shornick JK, Black MM. Secondary autoimmune diseases in herpes gestationis (pemphigoid gestationis). J Am Acad Dermatol 1992;26:563-6.

3. Dupont C. Herpes gestationis with hydatidiform mole. Trans St Johns Hosp Dermatol Soc 1974;60:103

4. Tindall JG, Rea TH, Shulman I, et al. Herpes gestationis in association with a hydatidiform mole. Immunopathologic studies. Arch Dermatol 1981;117:510-12.

5. Tillman WG. Herpes gestationis with hydatidiform mole and chorion epithelioma. Br Med J 1950:1:1471.

6. Holmes RC, Black MM, Dann J, et al. A comparative study of toxic erythema of pregnancy and herpes gestationis. Br J Dermatol 1982;106:499-510.

7. Morrison LH, Labib RS, Zone JJ, et al. Herpes gestationis autoantibodies recognize a 180-kD human epidermal antigen. J Clin Invest 1988;81:2023-6.

8. Kelly SE, Bhogal BS, Wojnarowska F, et al. Western blot analysis of the antigen in pemphigoid gestationis. Br J Dermatol 1990;122:445-9.

9. Chimanovitch I, Schmidt E, Messer G, et al. IgG1 and IgG3 are the major immunoglobulin subclasses targeting epitopes within the NC16A domain of BP180 in pemphigoid gestationis. J Invest Dermatol 1999;113:140-2.

10. Jenkins RE, Hern S, Black MM. Clinical features and management of 87 patients with pemphigoid gestationis. Clin Exp Dermatol 1999;24:255-9. 


\section{BMJ Case Reports}

11. Castro LA, Lundell RB, Krause PK, et al. Clinical experience in pemphigoid gestationis: report of 10 cases. J Am Acad Dermatol 2006;55:823-8.

12. Shimanovich I, Bröcker EB, Zillikens D. Pemphigoid gestationis: new insights into the pathogenesis lead to novel diagnostic tools. BJOG 2002:109:970-6.

13. Shornick JK. Dermatoses of pregnancy. Semin Cutan Med Surg 1998;17:172-81.

14. Al-Fouzan AW, Galadari I, Oumeish I, et al. Herpes gestationis (Pemphigoid gestationis). Clin Dermatol 2006;24:109-12.

15. Wollina U, Degen KW, Konrad H, et al. Itching stretch marks and bullous lesions in a pregnant woman. Int J Dermatol 2004;43:752-4.

16. Sitaru C, Powell J, Messer G, et al. Immunoblotting and enzyme-linked immunosorbent assay for the diagnosis of pemphigoid gestationis. Obstet Gynecol 2004;103:757-63.

17. Lawley TJ, Stingl G, Katz SI. Fetal and maternal risk factors in herpes gestationis. Arch Dermatol 1978;114:552-5.

18. Katz SI, Hertz KC, Yaoita H. Herpes gestationis. Immunopathology and characterization of the HG factor. J Clin Invest 1976;57:1434-41.

19. Jordon RE, Heine KG, Tappeiner G, et al. The immunopathology of herpes gestationis. Immunofluorescence studies and characterization of "HG factor". J Clin Invest 1976;57:1426-31.

20. Carruthers JA, Ewins AR. Herpes gestationis: studies on the binding characteristics, activity and pathogenetic significance of the complementfixing factor. Clin Exp Immunol 1978;31:38-44.

21. Provost TT, Tomasi TB Jr. Evidence for complement activation via the alternate pathway in skin diseases, I. Herpes gestationis, systemic lupus erythematosus, and bullous pemphigoid. J Clin Invest 1973;52:1779-87.

22. Scheman AJ, Hordinsky MD, Groth DW, et al. Evidence for eosinophil degranulation in the pathogenesis of herpes gestationis. Arch Dermatol 1989; 125:1079-83.

23. Carruthers JA, Black MM, Ramnarain N. Immunopathological studies in herpes gestationis. Br J Dermatol 1977;96:35-43.

24. Shornick JK, Stastny P, Gilliam JN. High frequency of histocompatibility antigens HLA-DR3 and DR4 in herpes gestations. J Clin Invest 1981;68:553-5.
25. Rodrigues Cdos S, Filipe P, Solana Mdel M, et al. Persistent herpes gestationis treated with high-dose intravenous immunoglobulin. Acta Derm Venereol 2007;87:184-6

26. Vaughan Jones SA, Hern S, Nelson-Piercy C, et al. A prospective study of 200 women with dermatoses of pregnancy correlating clinical findings with hormonal and immunopathological profiles. Br J Dermatol 1999;141:71-81.

27. Jolles $\mathbf{S}$. A review of high-dose intravenous immunoglobulin (hdIVIg) in the treatment of the autoimmune blistering disorders. Clin Exp Dermatol 2001;26:127-31.

28. Hern S, Harman K, Bhogal BS, et al. A severe persistent case of pemphigoid gestationis treated with intravenous immunoglobulins and cyclosporin. Clin Exp Dermatol 1998;23:185-8.

29. Van de Wiel A, Hart HC, Flinterman J, et al. Plasma exchange in herpes gestationis. Br Med J 1980;281:1041-2.

30. Lynch FW, Albrecht RJ. Homonal factors in herpes gestationis. Arch Dermatol 1966;93:446-7.

31. Hönigsmann H, Stingl G, Holubar K, et al. Herpes gestationis: fine structural pattern of immunoglobulin deposits in the skin in vivo. J Invest Dermatol 1976;66:389-92.

32. Lin MS, Arteaga LA, Diaz LA. Herpes gestationis. Clin Dermatol 2001;19:697-702.

33. Chorzelski TP, Jablonska S, Beutner EH, et al. Herpes gestations with identical lesions in the newborn. Passive transfer of the disease? Arch Dermatol 1976;112:1129-31.

34. Katz A, Minto J0, Toole JW, et al. Immunopathologic study of herpes gestationis in mother and infant. Arch Dermatol 1977;113:1069-72.

35. Faiz SA, Nainar SI, Addar MH. Herpes gestationis. Saudi Med 2004;25:792-4.

36. Hocking DR. Neonatal haemolytic disease due to dapsone. Med J Aust 1968;119:299-301.

37. Holmes RC, Black MM. The fetal prognosis in pemphigoid gestationis (herpes gestationis). Br J Dermatol 1984;110:67-72.

38. Jenkins RE, Shornick JK, Black MM. Pemphigoid gestationis. J Eur Acad Dermatol Venereol 1993;2:163-73.

39. Shornick JK, Black MM. Fetal risks in herpes gestationis. J Am Acad Dermatol 1992;26:63-8.

This pdf has been created automatically from the final edited text and images.

Copyright 2011 BMJ Publishing Group. All rights reserved. For permission to reuse any of this content visit http://group.bmj.com/group/rights-licensing/permissions.

BMJ Case Report Fellows may re-use this article for personal use and teaching without any further permission

Please cite this article as follows (you will need to access the article online to obtain the date of publication).

Lardenoije CMJG, van de Water M, Mertens HJMM, Gondrie ETCM. Pemphigoid gestationis. BMJ Case Reports 2011;10.1136/bcr.01.2010.2623, date of publication

Become a Fellow of BMJ Case Reports today and you can:

- Submit as many cases as you like

- Enjoy fast sympathetic peer review and rapid publication of accepted articles

- Access all the published articles

- Re-use any of the published material for personal use and teaching without further permission

For information on Institutional Fellowships contact consortiasales@bmjgroup.com

Visit casereports.bmj.com for more articles like this and to become a Fellow 